TRANSACTIONS OF THE

AMERICAN MATHEMATICAL SOCIETY

Volume 354, Number 3, Pages 993-1005

S 0002-9947(01)02870-7

Article electronically published on August 21, 2001

\title{
A DIMENSION INEQUALITY FOR COHEN-MACAULAY RINGS
}

\author{
SEAN SATHER-WAGSTAFF
}

\begin{abstract}
The recent work of Kurano and Roberts on Serre's positivity conjecture suggests the following dimension inequality: for prime ideals $\mathfrak{p}$ and $\mathfrak{q}$ in a local, Cohen-Macaulay ring $(A, \mathfrak{n})$ such that $e\left(A_{\mathfrak{p}}\right)=e(A)$ we have $\operatorname{dim}(A / \mathfrak{p})+\operatorname{dim}(A / \mathfrak{q}) \leq \operatorname{dim}(A)$. We establish this dimension inequality for excellent, local, Cohen-Macaulay rings which contain a field, for certain lowdimensional cases and when $R / \mathfrak{p}$ is regular.
\end{abstract}

\section{INTRODUCTION}

Let $(R, \mathfrak{m})$ be a local Noetherian ring of dimension $d$, and let $M$ and $N$ be finitely generated $R$-modules such that $M$ has finite projective dimension and $M \otimes_{R} N$ is a module of finite length. Serre [13] defined the intersection multiplicity of $M$ and $N$ to be

$$
\chi(M, N)=\sum_{i=0}^{n}(-1)^{i} \text { length }\left(\operatorname{Tor}_{i}^{R}(M, N)\right)
$$

and conjectured that $\chi(M, N)$ satisfies the following properties when $R$ is regular:

1. $\operatorname{dim}(M)+\operatorname{dim}(N) \leq \operatorname{dim}(R)$.

2. (Nonnegativity) $\chi(M, N) \geq 0$.

3. (Vanishing) If $\operatorname{dim}(M)+\operatorname{dim}(N)<\operatorname{dim}(R)$, then $\chi(M, N)=0$.

4. (Positivity) If $\operatorname{dim}(M)+\operatorname{dim}(N)=\operatorname{dim}(R)$, then $\chi(M, N)>0$.

Serre was able to verify the first statement for any regular local ring and the others in the case when $R$ is unramified. Since $\chi(M, N)$ has many of the characteristics we desire from an intersection multiplicity (for example, Bézout's Theorem holds), it was reasonable to suppose that these further properties are satisfied over an arbitrary regular local ring. The results were left unproved for ramified rings.

The vanishing conjecture was proved about ten years ago by Gillet and Soulé [3] and independently by Roberts [11] using $K$-theoretic methods. Gabber proved the nonnegativity conjecture recently [1, 6, 12, using a theorem of de Jong [2]. Kurano and Roberts have proved the following using methods introduced by Gabber.

Theorem 1.1 (7] Theorem 3.2). Assume that $(R, \mathfrak{m})$ is a regular local ring which either contains a field or is ramified. Also, assume that $\mathfrak{p}$ and $\mathfrak{q}$ are prime ideals in $R$ such that $\sqrt{\mathfrak{p}+\mathfrak{q}}=\mathfrak{m}$ and $\operatorname{dim}(R / \mathfrak{p})+\operatorname{dim}(R / \mathfrak{q})=\operatorname{dim} R$. If $\chi(R / \mathfrak{p}, R / \mathfrak{q})>0$ then

$$
\mathfrak{p}^{(n)} \cap \mathfrak{q} \subseteq \mathfrak{m}^{n+1} \quad \text { for all } n>0 .
$$

Received by the editors December 20, 1999 and, in revised form, March 1, 2000.

2000 Mathematics Subject Classification. Primary 13H15, 13C15; Secondary 13H05, 13D22.

Key words and phrases. Intersection dimension, intersection multiplicities, multiplicities. 
As a result, they conjectured that (1.1) should hold for all regular local rings. More specifically,

Conjecture 1.2. Assume that $(R, \mathfrak{m})$ is a regular local ring and that $\mathfrak{p}$ and $\mathfrak{q}$ are prime ideals in $R$ such that $\sqrt{\mathfrak{p}+\mathfrak{q}}=\mathfrak{m}$ and $\operatorname{dim}(R / \mathfrak{p})+\operatorname{dim}(R / \mathfrak{q})=\operatorname{dim} R$. Then $\mathfrak{p}^{(n)} \cap \mathfrak{q} \subseteq \mathfrak{m}^{n+1}$ for all $n>0$.

We study Conjecture 1.2, as a verification of this conjecture could introduce new tools to apply to the positivity conjecture.

For any local ring $(A, \mathfrak{n})$ let $e(A)$ denote the Samuel multiplicity of $A$ with respect to $\mathfrak{n}$ (see Section 2 for the precise definition). It is straightforward to verify that, if $R$ is a regular local ring with prime ideal $\mathfrak{p}$ and $0 \neq f \in \mathfrak{p}$, then $e\left(R_{\mathfrak{p}} /(f)\right)=m$ if and only if $f \in \mathfrak{p}^{(m)} \backslash \mathfrak{p}^{(m+1)}$. Thus, Conjecture 1.2 may be rephrased as the following.

Conjecture $1.2^{\prime}$. Assume that $(R, \mathfrak{m})$ is a regular local ring and that $\mathfrak{p}$ and $\mathfrak{q}$ are prime ideals in $R$ such that $\mathfrak{p}+\mathfrak{q}$ is $\mathfrak{m}$-primary. If there exists $0 \neq f \in \mathfrak{p} \cap \mathfrak{q}$ such that $e\left(R_{\mathfrak{p}} /(f)\right)=e(R /(f))$, then $\operatorname{dim}(R / \mathfrak{p})+\operatorname{dim}(R / \mathfrak{q}) \leq \operatorname{dim}(R)-1$.

Conjecture 1.2 motivates the following generalization.

Conjecture 1.3. Assume that $(R, \mathfrak{m})$ is a quasi-unmixed local ring and that $\mathfrak{p}$ and $\mathfrak{q}$ are prime ideals in $R$ such that $\sqrt{\mathfrak{p}+\mathfrak{q}}=\mathfrak{m}$ and $e\left(R_{\mathfrak{p}}\right)=e(R)$. Then $\operatorname{dim}(R / \mathfrak{p})+$ $\operatorname{dim}(R / \mathfrak{q}) \leq \operatorname{dim}(R)$.

Below, we present examples to show that our assumptions in Conjectures 1.2 and 1.3 are necessary.

As we noted above, Serre proved this conjecture in the case where $R$ is regular (where the condition $e\left(R_{\mathfrak{p}}\right)=e(R)$ holds automatically). The following is a famous conjecture which is rather similar to Conjecture 1.3.

Conjecture 1.4 (Peskine and Szpiro [10]). Assume that $(R, \mathfrak{m})$ is a local ring and $\mathfrak{p}$ and $\mathfrak{q}$ are prime ideals in $R$ such that $\mathfrak{p}$ has finite projective dimension and $\sqrt{\mathfrak{p}+\mathfrak{q}}=\mathfrak{m}$. Then $\operatorname{dim}(R / \mathfrak{p})+\operatorname{dim}(R / \mathfrak{q}) \leq \operatorname{dim}(R)$.

We discuss connections between Conjectures 1.3 and 1.4 below.

In Section 2 we prove Conjecture 1.3 in the case where $R$ is excellent and contains a field. In Section 3 we establish the Conjecture 1.3 for some low-dimensional cases and for the case where $R / \mathfrak{p}$ is regular. In Section 4 we present a number of examples.

I would like to express my gratitude to P. Roberts, A. Singh and the referee for their comments and suggestions.

\section{The Equicharacteristic Case}

All rings are assumed to be commutative and Noetherian with identity, and all modules are assumed to be unital.

The goal of this section is to prove the following theorem.

Theorem 2.1. Assume that $(R, \mathfrak{m})$ is an excellent local Cohen-Macaulay ring which contains a field. Also, assume that $P$ and $Q$ are prime ideals of $R$ such that $\sqrt{P+Q}=\mathfrak{m}$ and $e\left(R_{P}\right)=e(R)$. Then $\operatorname{dim}(R / P)+\operatorname{dim}(R / Q) \leq \operatorname{dim}(R)$.

We accomplish this in steps. First, we fix some notation and definitions and prove Theorem 2.1] in the case where $R$ is complete with infinite residue field. Then, we 
establish some results on the behavior of the Samuel multiplicity and reduce the case where $R$ is excellent to the the case where our ring is complete.

The Samuel multiplicity shall play a central role in our work. For the sake of clarity we specify which multiplicity we are considering. Assume that $(R, \mathfrak{m})$ is a local ring of dimension $d$ with an ideal $\mathfrak{a}$ such that $\sqrt{\mathfrak{a}}=\mathfrak{m}$. For $n \gg 0$ the Hilbert function $H_{\mathfrak{a}}(n)=\operatorname{len}\left(R / \mathfrak{a}^{n+1}\right)$ is a polynomial in $n$ of degree $d$ with rational coefficients. If $e_{d}$ is the leading coefficient of this polynomial, then the Samuel multiplicity of $\mathfrak{a}$ on $R$ is $e(\mathfrak{a}, R)=d ! e_{d}$. We denote $e(\mathfrak{m}, R)$ by $e(R)$. Recall that the Samuel multiplicity satisfies the Associativity Formula

$$
e(R)=\sum_{\mathfrak{p}} \operatorname{len}\left(R_{\mathfrak{p}}\right) e(R / \mathfrak{p})
$$

where the sum is taken over all prime ideals $\mathfrak{p}$ of $R$ such that $\operatorname{dim}(R / \mathfrak{p})=d$.

We first prove Theorem 2.1 for the case where $R$ is a complete, Cohen-Macaulay ring with infinite residue field $K$. In this case we can find a system of parameters $y_{1}, \ldots, y_{n}$ which form part of a minimal set of generators of $\mathfrak{m}$ such that $\operatorname{len}\left(R /\left(y_{1}, \ldots, y_{n}\right)\right)=e(R)$ (see Matsumura 9] Theorem 14.14). Since $R$ is assumed complete, $R$ is a finite extension of the power series ring $K \llbracket Y_{1}, \ldots, Y_{n} \rrbracket$, and since $R$ is Cohen-Macaulay, it is a free module over this subring of rank $e(R)$.

The main result used in proving Theorem 2.1 is the following:

Theorem 2.2. Assume that $B$ is a Cohen-Macaulay ring and $(A, \mathfrak{m})$ a regular local subring such that $B$ is a finite free $A$-module. Assume that $P$ is a prime ideal of $B$ with $P \cap A=\mathfrak{p}$ and $e\left(B_{P}\right)=\operatorname{rank}_{A}(B)$. Then $P$ is the unique prime ideal of $B$ which contracts to $\mathfrak{p}$ in $A$, and $B_{P} / P_{P} \cong A_{\mathfrak{p}} / \mathfrak{p}_{\mathfrak{p}}$.

Proof. First, we reduce to the case where $\mathfrak{p}$ is the maximal ideal of $A$. Any localization of a Cohen-Macaulay ring is Cohen-Macaulay, so $B_{\mathfrak{p}}=B \otimes_{A} A_{\mathfrak{p}}$ is CohenMacaulay. The ring $A_{\mathfrak{p}}$ is regular and $A_{\mathfrak{p}}$ is a subring of $B_{\mathfrak{p}}$. If $r=\operatorname{rank}_{A}(B)$ then $B \cong A^{r}$ and so $B_{\mathfrak{p}} \cong A_{\mathfrak{p}}^{r}$ is finite and free over $A_{\mathfrak{p}}$. Furthermore, $P_{\mathfrak{p}}$ is a prime ideal of $B_{\mathfrak{p}}$ which contracts to the maximal ideal of $A_{\mathfrak{p}}$ and $e\left(\left(B_{\mathfrak{p}}\right)_{P}\right)=e\left(B_{P}\right)=$ $\operatorname{rank}_{A}(B)=r=\operatorname{rank}_{A_{\mathfrak{p}}}\left(B_{\mathfrak{p}}\right)$. Thus, the rings $B_{\mathfrak{p}}$ and $A_{\mathfrak{p}}$ satisfy the hypotheses of the theorem and we may assume that $P \cap A=\mathfrak{m}$.

By the finiteness of the extension $A \rightarrow B$, the prime ideal $P$ is maximal. The ring $B / \mathfrak{m} B$ is Artinian because any prime ideal of $B$ which contains $\mathfrak{m} B$ must contract to $\mathfrak{m}$ in $A$ and therefore must be maximal. In particular, a regular system of parameters of $A$ passes to a system of parameters of $B_{P}$ (and since $B_{P}$ is CohenMacaulay, a maximal $B_{P}$-regular sequence). Let $K=A / \mathfrak{m}, L=B / P$ and $\hat{P}=$ $P / \mathfrak{m} B$. By the finiteness of the extension $A \rightarrow B$, the extension $K \rightarrow L$ is finite. Since $B / \mathfrak{m} B$ is a finite-dimensional vector space over $K, B / \mathfrak{m} B$ has finite length as an $A$-module. By the structure theorem for Artinian rings, $B / \mathfrak{m} B$ has a finite number of maximal ideals, $\hat{P}=\hat{P}_{1}, \hat{P}_{2}, \ldots, \hat{P}_{l}$, and $B / \mathfrak{m} B \cong \prod_{i}(B / \mathfrak{m} B)_{\hat{P}_{i}}$. In particular, each $(B / \mathfrak{m} B)_{\hat{P}_{i}}$ is finitely generated over $A$ and has finite length as an $A$-module. It is straightforward to verify that

$$
\operatorname{len}_{B_{P}}\left(B_{P} / \mathfrak{m} B_{P}\right)=\operatorname{len}_{A}\left(B_{P} / \mathfrak{m} B_{P}\right) / \operatorname{dim}_{K}(L) .
$$


By [9] Theorem $14.9, e\left(B_{P}\right) \leq e\left(B_{P} / \mathfrak{m} B_{P}\right)$ since $\mathfrak{m}$ is generated by a $B_{P}$-regular sequence. Our assumptions imply that

$$
\begin{aligned}
\sum_{i} \operatorname{dim}_{K}\left((B / \mathfrak{m} B)_{\hat{P}_{i}}\right) & =\operatorname{dim}_{K}(B / \mathfrak{m} B)=\operatorname{rank}_{A}(B)=e\left(B_{P}\right) \leq e\left(B_{P} / \mathfrak{m} B_{P}\right) \\
& =\operatorname{len}_{B_{P}}\left(B_{P} / \mathfrak{m} B_{P}\right)=\operatorname{len}_{A}\left(B_{P} / \mathfrak{m} B_{P}\right) / \operatorname{dim}_{K}(L) \\
& =\operatorname{dim}_{K}\left(B_{P} / \mathfrak{m} B_{P}\right) / \operatorname{dim}_{K}(L) \leq \operatorname{dim}_{K}\left(B_{P} / \mathfrak{m} B_{P}\right) \\
& \leq \sum_{i} \operatorname{dim}_{K}\left((B / \mathfrak{m} B)_{\hat{P}_{i}}\right)
\end{aligned}
$$

so we must have equality. This can only happen if (i) $\hat{P}$ is the unique prime ideal of $B / \mathfrak{m} B$ and (ii) $\operatorname{dim}_{K}(L)=1$. These are the desired results.

Proof of Theorem 2.1 when $R$ is complete with infinite residue field. As noted previously, we may choose a system of parameters $y_{1}, \ldots, y_{n}$ of $R$ such that (i) the $y_{i}$ form part of a minimal generating set of $\mathfrak{m}$ and (ii) $e(R)=\operatorname{len}\left(R /\left(y_{1}, \ldots, y_{d}\right)\right)$. Fix $z_{1}, \ldots, z_{q} \in \mathfrak{m}$ such that $y_{1}, \ldots, y_{n}, z_{1}, \ldots, z_{q}$ form a minimal generating set for $\mathfrak{m}$. The natural map $K \llbracket Y_{1}, \ldots, Y_{n} \rrbracket \rightarrow R$ given by $Y_{i} \mapsto y_{i}$ is injective since $R$ is complete, and $R$ is module finite over $A=K \llbracket Y_{1}, \ldots, Y_{n} \rrbracket$ (cf., 9 929 ), and the fact that $R$ is local Cohen-Macaulay implies that $R$ is free over $A$ of finite rank $r$. Furthermore, the natural map $\rho: K \llbracket Y_{1}, \ldots, Y_{n}, Z_{1}, \ldots, Z_{q} \rrbracket \rightarrow R$ given by $Y_{i} \mapsto y_{i}$ and $Z_{j} \mapsto z_{j}$ is surjective. Let $A^{\prime}=K \llbracket Y_{1}, \ldots, Y_{n}, Z_{1}, \ldots, Z_{q} \rrbracket$ with maximal ideal $\mathfrak{m}^{\prime}$ and $I=\operatorname{ker}(\rho)$. The constructions show that we have a natural commuting diagram:

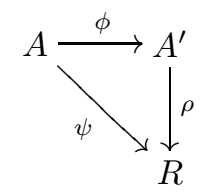

Let $\mathfrak{p}=P \cap A$. Since the extension $A \rightarrow R$ is finite and free, both the going-up and going-down properties hold so that ht $(\mathfrak{p})=\mathrm{ht}(P)$ and $\operatorname{dim}(A / \mathfrak{p})=\operatorname{dim}(R / P)$. If we can show that $\sqrt{\mathfrak{p} A^{\prime}+\rho^{-1}(Q)}=\mathfrak{m}^{\prime}$, then it follows that

$$
\begin{aligned}
\operatorname{dim}(R / P)+\operatorname{dim}(R / Q) & =\operatorname{dim}(A / \mathfrak{p})+\operatorname{dim}\left(A^{\prime} / \rho^{-1}(Q)\right) \\
& =\operatorname{dim}\left(A^{\prime} / \mathfrak{p} A^{\prime}\right)-q+\operatorname{dim}\left(A^{\prime} / \rho^{-1}(Q)\right) \\
& \leq \operatorname{dim}\left(A^{\prime}\right)-q=n=\operatorname{dim}(R)
\end{aligned}
$$

where the final inequality follows from the fact that Theorem 2.1 holds for regular local rings.

For an ideal $\mathfrak{a}$ of $A^{\prime}$, let $Z(\mathfrak{a}) \subseteq \operatorname{Spec}\left(A^{\prime}\right)$ denote the closed subscheme determined by $\mathfrak{a}$. In order to show that $\sqrt{\mathfrak{p} A^{\prime}+\rho^{-1}(Q)}=\mathfrak{m}^{\prime}$, it suffices to show that

$$
Z\left(\mathfrak{p} A^{\prime}+\rho^{-1}(Q)\right)=Z\left(\rho^{-1}(P)+\rho^{-1}(Q)\right)
$$

as the surjectivity of $\rho$ implies that $\sqrt{\rho^{-1}(P)+\rho^{-1}(Q)}=\mathfrak{m}^{\prime}$. Since $\mathfrak{p} A^{\prime}+\rho^{-1}(Q) \subseteq$ $\rho^{-1}(P)+\rho^{-1}(Q)$, the inclusion $Z\left(\mathfrak{p} A^{\prime}+\rho^{-1}(Q)\right) \supseteq Z\left(\rho^{-1}(P)+\rho^{-1}(Q)\right)$ is clear. For the other inclusion we note that, since $I \subseteq \rho^{-1}(Q)$,

$$
\mathfrak{p} A^{\prime}+\rho^{-1}(Q)=\left(\mathfrak{p} A^{\prime}+I\right)+\rho^{-1}(Q)
$$


so that

$$
Z\left(\mathfrak{p} A^{\prime}+\rho^{-1}(Q)\right)=Z\left(\left(\mathfrak{p} A^{\prime}+I\right)+\rho^{-1}(Q)\right)=Z\left(\mathfrak{p} A^{\prime}+I\right) \cap Z\left(\rho^{-1}(Q)\right) .
$$

Since $Z\left(\rho^{-1}(P)+\rho^{-1}(Q)\right)=Z\left(\rho^{-1}(P)\right) \cap Z\left(\rho^{-1}(Q)\right)$ it then suffices to show that $Z\left(\mathfrak{p} A^{\prime}+I\right) \subseteq Z\left(\rho^{-1}(P)\right)$. It suffices to show that $\rho^{-1}(P)$ determines the unique minimal prime ideal of $A^{\prime} /\left(\mathfrak{p} A^{\prime}+I\right)$. By our commuting diagram, the (minimal) primes of $\mathfrak{p} A^{\prime}+I$ are in bijection with the (minimal) primes of $\mathfrak{p} R=\rho\left(\mathfrak{p} A^{\prime}+I\right)$. Thus, it suffices to show that $P$ is the unique minimal prime of $\mathfrak{p} R$. By Lemma [2.3 below, every minimal prime $P^{\prime}$ of $\mathfrak{p} R$ contracts to $\mathfrak{p}$ in $A$, so it suffices to show that $P$ is the unique prime ideal of $R$ such that $P \cap A=\mathfrak{p}$. By assumption $P \cap A=\mathfrak{p}$ and $e\left(R_{P}\right)=e(R)$. So, if we can show that $e(R)=\operatorname{rank}_{A}(R)$, then Theorem 2.2 supplies the necessary uniqueness. Our assumptions imply that

$$
\begin{aligned}
e(R) & =\operatorname{len}(R /(\mathbf{y}) R)=\operatorname{dim}_{K}(R /(\mathbf{y}) R)=\operatorname{dim}_{K}\left(R \otimes_{A} A /(\mathbf{y}) A\right) \\
& =\operatorname{dim}_{K}\left(R \otimes_{A} K\right)=\operatorname{dim}_{K}\left(A^{r} \otimes_{A} K\right)=\operatorname{dim}_{K}\left(K^{r}\right)=r=\operatorname{rank}_{A}(R)
\end{aligned}
$$

and this completes the proof.

Lemma 2.3. Assume that $R \rightarrow \tilde{R}$ is a flat local homomorphism of local rings $(R, \mathfrak{m})$ and $(\tilde{R}, \tilde{m})$.

1. If $\mathfrak{m} \tilde{R}$ is $\tilde{\mathfrak{m}}$-primary, then $\operatorname{dim}(\tilde{R})=\operatorname{dim}(R)$.

2. If $\mathfrak{m} \tilde{R}$ is $\tilde{\mathfrak{m}}$-primary and $R$ is Cohen-Macaulay, then $\tilde{R}$ is Cohen-Macaulay.

3. If $\mathfrak{p}$ is a prime ideal of $R$ and $P$ is a prime ideal of $\tilde{R}$ which is minimal over $\mathfrak{p} \tilde{R}$, then $P \cap R=\mathfrak{p}$ and ht $(P)=\mathrm{ht}(\mathfrak{p})$.

Proof. 1. Let $d=\operatorname{dim}(R)$. By flatness, the extension $R \rightarrow \tilde{R}$ satisfies the goingdown property. In particular, $d \leq \operatorname{dim}(\tilde{R})$. Let $x_{1}, \ldots, x_{d} \in \mathfrak{m}$ be a system of parameters for $R$ and let $\mathfrak{a}=(\mathbf{x}) R$. Then $\sqrt{\mathfrak{a}}=\mathfrak{m}$ so that $\sqrt{\mathfrak{a} \tilde{R}}=\tilde{\mathfrak{m}}$. Thus, $\mathfrak{a} \tilde{R}$ is $\tilde{\mathfrak{m}}$-primary and generated by $d$ elements, so that $d \geq \operatorname{dim}(\tilde{R})$.

2. The system of parameters $\mathbf{x}$ is a regular sequence on $R$ of length $d$. By flatness $\mathbf{x}$ is a regular sequence on $\tilde{R}$ of length $\operatorname{dim}(\tilde{R})$.

3. Suppose that $P \cap R \neq \mathfrak{p}$. Since $P \cap R \supsetneq \mathfrak{p}$, the going-down property implies that $P$ contains a prime ideal $Q$ which contracts to $\mathfrak{p}$. But then $\mathfrak{p} \tilde{R} \subseteq Q \subsetneq P$, contradicting the minimality of $P$. The fact that ht $(P)=\mathrm{ht}(\mathfrak{p})$ is proved in [4] Lemma 18.10 .

The remainder of this section is devoted to proving that we can reduce Theorem 2.1 to the case where $R$ is complete.

For technical reasons, we must restrict our attention to certain classes of rings. We recall the definitions here. A ring $R$ is equidimensional if $\operatorname{dim}(R / \mathfrak{p})=\operatorname{dim}(R)$ for all minimal prime ideals $\mathfrak{p}$ of $R$. A local ring $R$ is quasi-unmixed if its completion is equidimensional. A ring $R$ is Nagata if, for every prime ideal $\mathfrak{p}$ of $R$ and every finite extension field $L$ of the quotient field of $R / \mathfrak{p}$, the integral closure of $R / \mathfrak{p}$ in $L$ is module-finite over $R / \mathfrak{p}$.

Every Cohen-Macaulay ring is equidimensional. Every excellent ring 11 is Nagata. Furthermore, the class of Nagata rings is closed under finite ring extensions, localizations and quotients. Also, every localization of a ring of polynomials over a

\footnotetext{
${ }^{1}$ For a complete definition of "excellent", see Matsumura [9] Section 32. It is important to note that the class of excellent rings contains all fields, complete local rings and the ring of integers, and is closed under finite ring extensions, localizations and quotients.
} 
quasi-unmixed ring is quasi-unmixed. Furthermore, a quasi-unmixed local ring is equidimensional and universally catenary (see Hermann, Ikeda and Orbanz [4] Section 18). One of the advantages of working with Nagata rings is that multiplicities are well-behaved with respect to localization. The following is a special case of a theorem of Lech [8].

Lemma 2.4. Assume that $(R, \mathfrak{m})$ is a local, quasi-unmixed, Nagata ring with prime ideal $\mathfrak{p}$. Then $e\left(R_{\mathfrak{p}}\right) \leq e(R)$.

Proof. Because $R$ is equidimensional and catenary, ht $\mathfrak{p}+\operatorname{dim}(R / \mathfrak{p})=\operatorname{dim}(R)$. Because $R$ is Nagata, for every pair of primes $\mathfrak{q} \subset \mathfrak{q}^{\prime}$, the integral closure of $R_{\mathfrak{q}^{\prime}} / \mathfrak{q} R_{\mathfrak{q}^{\prime}}$ is module-finite over $R_{\mathfrak{q}^{\prime}} / \mathfrak{q} R_{\mathfrak{q}^{\prime}}$. As noted by Lech ([8] comments following Theorem 1) this now implies that $e\left(R_{\mathfrak{p}}\right) \leq e(R)$.

Lech conjectured that Lemma 2.4 should hold for any local, equidimensional ring. Below, we indicate an example which shows that we must assume that $R$ is at least equidimensional in order for the inequality $e\left(R_{\mathfrak{p}}\right) \leq e(R)$ to hold for all prime ideals $\mathfrak{p}$ of $R$.

In the following two lemmas, we collect facts that show that multiplicities do not change under certain ring extensions. We will use these facts to reduce our general question to the situation where $R$ is complete with infinite residue field and $R / \mathfrak{p}$ is normal. For a finitely generated module $M$, let $\mu(M)$ denote the minimal number of generators of $M$.

Lemma 2.5. Assume that $R \rightarrow \tilde{R}$ is a flat local homomorphism of Noetherian local rings $(R, \mathfrak{m})$ and $(\tilde{R}, \tilde{m})$ and that $\mathfrak{a}$ is an ideal of $R$.

1. $\mu(\mathfrak{a})=\mu(\mathfrak{a} \tilde{R})$.

2. If, in addition, $\mathfrak{m} \tilde{R}=\tilde{\mathfrak{m}}$ then $\operatorname{len}_{R}\left(\mathfrak{m}^{n} / \mathfrak{m}^{n+1}\right)=\operatorname{len}_{\tilde{R}}\left(\tilde{\mathfrak{m}}^{n} / \tilde{\mathfrak{m}}^{n+1}\right)$. In particular, $e(R)=e(\tilde{R})$.

Proof. Part 1 is proved by Herzog [5] Lemma 2.3. Part 2 follows from part 1 by the following computation

$$
\operatorname{len}_{R}\left(\mathfrak{m}^{n} / \mathfrak{m}^{n+1}\right)=\mu\left(\mathfrak{m}^{n}\right)=\mu\left(\mathfrak{m}^{n} \tilde{R}\right)=\mu\left(\tilde{\mathfrak{m}}^{n}\right)=\operatorname{len}_{\tilde{R}}\left(\tilde{\mathfrak{m}}^{n} / \tilde{\mathfrak{m}}^{n+1}\right)
$$

and the definitions of $e(R)$ and $e(\tilde{R})$.

Lemma 2.6. Let $(R, \mathfrak{m})$ be a quasi-unmixed local ring and $S=R\left[X_{1}, \ldots, X_{n}\right]$ a polynomial ring over $R$. Let $P$ be a prime ideal of $S$ and $\mathfrak{p}=P \cap R$. Then $e\left(R_{\mathfrak{p}}\right)=e\left(S_{P}\right)$.

Proof. By induction, we may assume that $n=1$, and the result follows in this case by [8] Lemma 2.

The following theorem allows us to reduce Conjecture 1.3 to the case where the quotient $R / \mathfrak{p}$ is a normal domain. This will be a key step in our reduction of Theorem 2.1 to the case where $R$ is complete.

Theorem 2.7. Let $(R, \mathfrak{m})$ be a Nagata, Cohen-Macaulay local ring, and suppose that, for every ring $(S, M)$ which is a localization at a maximal ideal of a polynomial ring over $R$, the following holds: for all prime ideals $P$ and $Q$ such that $\sqrt{P+Q}=$ $M, e\left(S_{P}\right)=e(S)$ and $S / P$ is a normal domain,

$$
\operatorname{dim}(S / P)+\operatorname{dim}(S / Q) \leq \operatorname{dim}(S)
$$


Then, for all prime ideals $\mathfrak{p}$ and $\mathfrak{q}$ of $R$ such that $\sqrt{\mathfrak{p}+\mathfrak{q}}=\mathfrak{m}$ and $e\left(R_{\mathfrak{p}}\right)=e(R)$,

$$
\operatorname{dim}(R / \mathfrak{p})+\operatorname{dim}(R / \mathfrak{q}) \leq \operatorname{dim}(R) .
$$

Proof. Fix prime ideals $\mathfrak{p}$ and $\mathfrak{q}$ of $R$ such that $\sqrt{\mathfrak{p}+\mathfrak{q}}=\mathfrak{m}$ and $e\left(R_{\mathfrak{p}}\right)=e(R)$. Let $B$ be the integral closure of $R / \mathfrak{p}$. Since $R$ is Nagata, $B$ is module-finite over $R / \mathfrak{p}$ and in particular, $\operatorname{dim}(B)=\operatorname{dim}(R / \mathfrak{p})$. Because $B$ is module finite over $R / \mathfrak{p}$, there is a surjection $T=R\left[X_{1}, \ldots, X_{n}\right] \rightarrow B$. Let $K$ denote the kernel of this map so that $T / K=B$. The commutative diagram

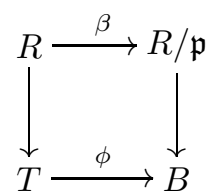

shows that $K \cap R=\mathfrak{p}$. Let $\mathfrak{n} \subset B$ be a maximal ideal. Since the extension $R / \mathfrak{p} \rightarrow B$ is finite, we know that $\mathfrak{n} \cap R / \mathfrak{p}$ is maximal in $R / \mathfrak{p}$, that is, $\mathfrak{n} \cap R / \mathfrak{p}=\mathfrak{m} / \mathfrak{p}$. Also, there are no prime ideals of $B$ which are properly contained in $\mathfrak{n}$ and contract to $\mathfrak{m} / \mathfrak{p}$ in $R / \mathfrak{p}$. It follows that $\sqrt{\mathfrak{q} B_{\mathfrak{n}}}=\sqrt{(\mathfrak{m} / \mathfrak{p}) B_{\mathfrak{n}}}=\mathfrak{n}_{\mathfrak{n}}$.

Let $N=\phi^{-1}(\mathfrak{n})$, so that $T / N \cong B / \mathfrak{n}$. Then, $K \subseteq N$ and since $\mathfrak{m}=\beta^{-1}(\mathfrak{n})=$ $R \cap \phi^{-1}(\mathfrak{n})=R \cap N$, we see that $L=\mathfrak{q} T \subseteq N$. We claim that $\sqrt{K_{N}+L_{N}}=N_{N}$. Since $K+L \supseteq K=\operatorname{ker}(\phi)$ we see that $K+L=\phi^{-1}(\phi(K+L))=\phi^{-1}(\mathfrak{q} B)$. Let $\phi$ denote the map $T_{N} \rightarrow B_{\mathfrak{n}}$. If $x \in N_{N}$, then $\phi(x) \in \mathfrak{n}$ so that for some $n$, $\phi\left(x^{n}\right)=\phi(x)^{n} \in \mathfrak{q} B_{\mathfrak{n}}$. Then $x^{n} \in \phi^{-1}\left(\mathfrak{q} B_{\mathfrak{n}}\right)=(K+L)_{N}$ so that $N_{N} \subseteq \sqrt{K_{N}+L_{N}}$ as desired.

By Lemma 2.6, we see that $e\left(T_{N}\right)=e(R)=e\left(R_{\mathfrak{p}}\right)=e\left(T_{K}\right)$. By construction, $T_{N} / K_{N}$ is a normal domain. Thus, if we know that

$$
\operatorname{dim}\left(T_{N} / K_{N}\right)+\operatorname{dim}\left(T_{N} / L_{N}\right) \leq \operatorname{dim}\left(T_{N}\right)
$$

then it follows that

$$
\begin{aligned}
\operatorname{dim}(R / \mathfrak{p})+\operatorname{dim}(R / \mathfrak{q}) & =\operatorname{dim}\left(T_{N} / K_{N}\right)+\operatorname{dim}\left(T_{N} / L_{N}\right)-n \\
& \leq \operatorname{dim}\left(T_{N}\right)-n=\operatorname{dim}(R)
\end{aligned}
$$

as desired. This gives the desired reduction.

It is clear that, in the statement of Theorem 2.7, "Cohen-Macaulay" may be replaced by either "quasi-unmixed", "Gorenstein" or "compete intersection (of codimension $c$ )". The same is true of the statement of Theorem 2.8 below. Below, we indicate an example which shows that we must assume that our ring is at least equidimensional for Conjecture 1.3 to hold.

The following theorem is the result which will allow us to reduce Conjecture 1.3 to the case where $R$ is complete.

Theorem 2.8. Let $(R, \mathfrak{m})$ be an excellent, Cohen-Macaulay local ring. Assume the following for every ring $(S, M)$ which is the localization of a polynomial ring over $R$ at a maximal ideal: for all prime ideals $\hat{P}, \hat{Q}$ of the completion $\hat{S}$ such that $\sqrt{\hat{P}+\hat{Q}}=\hat{M}, e\left(\hat{S}_{\hat{P}}\right)=e(\hat{S})$ and $\hat{S} / \hat{P}$ is a normal domain, we have

$$
\operatorname{dim}(\hat{S} / \hat{P})+\operatorname{dim}(\hat{S} / \hat{Q}) \leq \operatorname{dim}(\hat{S}) .
$$

Then, for all prime ideals $\mathfrak{p}$ and $\mathfrak{q}$ of $R$ such that $\sqrt{\mathfrak{p}+\mathfrak{q}}=\mathfrak{m}$ and $e\left(R_{\mathfrak{p}}\right)=e(R)$,

$$
\operatorname{dim}(R / \mathfrak{p})+\operatorname{dim}(R / \mathfrak{q}) \leq \operatorname{dim}(R)
$$


Proof. By Theorem 2.7 it suffices to show that for every ring $(S, M)$ which is a localization at a maximal ideal of a polynomial ring over $R$, the following holds: for all prime ideals $P$ and $Q$ such that $\sqrt{P+Q}=M, e\left(S_{P}\right)=e(S)$ and $S / P$ is a normal domain,

$$
\operatorname{dim}(S / P)+\operatorname{dim}(S / Q) \leq \operatorname{dim}(S)
$$

Let $S, M, P, Q$ satisfy these hypotheses. The ring $S$ is excellent, which implies that $S / P$ is also excellent (cf., 9] $\S 32$ ). By [9] Theorem $32.2, \widehat{S / P}=\hat{S} / P \hat{S}$ is normal, in particular, $\hat{P}=P \hat{S}$ is a prime ideal of $\hat{S}$ such that $\hat{S} / \hat{P}$ is a normal domain. Since the map $S \rightarrow \hat{S}$ is faithfully flat, $\hat{P} \cap S=P$. Thus, by Lemma 2.3, ht $(\hat{P})=\operatorname{ht}(P)$. By [4] Theorem $18.13(\mathrm{~d}), \operatorname{dim}(\hat{S} / \hat{P})=\operatorname{dim}(S / P)$. Let $\hat{Q}$ be a minimal prime ideal containing $Q \hat{S}$ such that $\operatorname{dim}(\hat{S} / \hat{Q})=\operatorname{dim}(\hat{S} / Q \hat{S})$. The extension $S_{P} \rightarrow \hat{S}_{\hat{P}}$ is faithfully flat and $P_{P} \hat{S}_{\hat{P}}=\hat{P}_{\hat{P}}$ so that, by Lemma 2.5

$$
e\left(\hat{S}_{\hat{P}}\right)=e\left(S_{P}\right)=e(S)=e(\hat{S})
$$

Thus, by assumption

$$
\operatorname{dim}(S / P)+\operatorname{dim}(S / Q)=\operatorname{dim}(\hat{S} / \hat{P})+\operatorname{dim}(\hat{S} / \hat{Q}) \leq \operatorname{dim}(\hat{S})=\operatorname{dim}(S)
$$

as desired.

Now, we complete the proof of Theorem 2.1 .

Proof of Theorem 2.1. If the residue field of $R$ is finite, let $X$ be an indeterminate over $R$ and $R(X)=R[X]_{\mathfrak{m}[X]}$ which is an excellent, Cohen-Macaulay local ring that contains a field. The prime ideals $P=\mathfrak{p} R(X)$ and $Q=\mathfrak{q} R(X)$ satisfy the following properties: $\sqrt{P+Q}=\mathfrak{m} R(X), R(X) / P=R / \mathfrak{p}(X), R(X) / Q=R / \mathfrak{q}(X)$ and (by Lemma 2.6) $e\left(R(X)_{P}\right)=e\left(R_{\mathfrak{p}}\right)=e(R)=e(R(X))$. Thus, if the theorem holds for $R(X)$, then

$$
\begin{aligned}
\operatorname{dim}(R / \mathfrak{p})+\operatorname{dim}(R / \mathfrak{q}) & =\operatorname{dim}(R(X) / P)+\operatorname{dim}(R(X) / Q) \\
& \leq \operatorname{dim}(R(X))=\operatorname{dim}(R)
\end{aligned}
$$

and the theorem holds for $R$. Since the residue field of $R(X)$ is $K(X)$, we may assume that the residue field of $R$ is infinite. By Theorems 2.7 and 2.8 we may pass to the completion of the localization of a polynomial ring over $R$. In particular, we may assume that $R$ is a complete local Cohen-Macaulay ring which contains a field, and by our previous remarks this completes the proof.

\section{Other CAses}

A number of low dimensional cases of Conjecture 1.3 can be dealt with rather easily using Lemma 2.4 and the Associativity Formula for multiplicities.

Proposition 3.1. Assume that $(R, \mathfrak{m})$ is an equidimensional local ring of dimension d with prime ideals $\mathfrak{p}$ and $\mathfrak{q}$ such that $\sqrt{\mathfrak{p}+\mathfrak{q}}=\mathfrak{m}$ and $e\left(R_{\mathfrak{p}}\right)=e(R)$.

1. If $\mathfrak{p}$ is a minimal prime ideal of $R$ then $\mathfrak{q}=\mathfrak{m}$.

2. If $\operatorname{dim}(R / \mathfrak{q})=1$ then $\operatorname{dim}(R / \mathfrak{p})<d$.

3. If $R$ is Nagata and $\mathfrak{q}$ is a minimal prime ideal of $R$ then $\mathfrak{p}=\mathfrak{m}$.

4. If $R$ is Nagata and $\operatorname{dim}(R / \mathfrak{p})=1$ then $\operatorname{dim}(R / \mathfrak{q})<d$.

In particular, Conjecture 1.3 holds when $R$ is Nagata and one of the following holds: $\operatorname{dim}(R / \mathfrak{p})=1, \operatorname{dim}(R / \mathfrak{p})=d, \operatorname{dim}(R / \mathfrak{q})=1$, or $\operatorname{dim}(R / \mathfrak{q})=d$ 
Proof. We prove part 1 here. The remaining parts are proved similarly. Our assumptions imply that

$$
\operatorname{len}\left(R_{\mathfrak{p}}\right)=e\left(R_{\mathfrak{p}}\right)=e(R)
$$

and the Associativity Formula implies that

$$
\operatorname{len}\left(R_{\mathfrak{p}}\right)=e(R)=\sum_{\mathfrak{r}} \operatorname{len}\left(R_{\mathfrak{r}}\right) e(R / \mathfrak{r})
$$

where the sum is taken over all prime ideals $\mathfrak{r}$ of $R \operatorname{such}$ that $\operatorname{dim}(R / \mathfrak{r})=d$. Since each $e(R / \mathfrak{r})>0$, it follows that $\operatorname{len}\left(R_{\mathfrak{p}}\right) e(R / \mathfrak{p})$ is the only term in the sum. Since $R$ is equidimensional, this implies that $\mathfrak{p}$ is the unique minimal prime ideal of $R$. In particular, $\mathfrak{q} \supseteq \sqrt{(0)}=\mathfrak{p}$. The fact that $\mathfrak{q}$ is prime implies that $\mathfrak{m}=\sqrt{\mathfrak{p}+\mathfrak{q}}=$ $\sqrt{\mathfrak{q}}=\mathfrak{q}$, as desired.

The proof of Proposition 3.1 shows that, for minimal prime ideals $\mathfrak{p}$, the assumption $e\left(R_{\mathfrak{p}}\right)=e(R)$ is quite strong. In fact, we have the following.

Proposition 3.2. Assume that $(R, \mathfrak{m})$ is an equidimensional local ring with minimal prime ideal $\mathfrak{p}$, and consider the following statements.

1. $e(R / \mathfrak{p})=1$.

2. $e\left(R_{\mathfrak{p}}\right)=e(R)$.

3. $\mathfrak{p}$ is the unique minimal prime of $R$.

(a) Any two of these conditions imply the third.

(b) If $R / \mathfrak{p}$ is unmixed (that is, if every associated prime $\mathfrak{p}^{\prime}$ of the completion $R^{\prime}$ of $R / \mathfrak{p}$ satisfies the condition $\left.\operatorname{dim}\left(R^{\prime} / \mathfrak{p}^{\prime}\right)=\operatorname{dim}\left(R^{\prime}\right)\right)$ then condition 1 may be replaced by " $R / \mathfrak{p}$ is regular".

Proof. (a) If $e(R / \mathfrak{p})=1$ and $e\left(R_{\mathfrak{p}}\right)=e(R)$, then

$$
\begin{aligned}
& \operatorname{len}\left(R_{\mathfrak{p}}\right)=e\left(R_{\mathfrak{p}}\right)=e(R)=\sum_{\mathfrak{r}} \operatorname{len}\left(R_{\mathfrak{r}}\right) e(R / \mathfrak{r}) \\
& \geq \operatorname{len}\left(R_{\mathfrak{p}}\right) e(R / \mathfrak{p})=\operatorname{len}\left(R_{\mathfrak{p}}\right)
\end{aligned}
$$

where the sum is taken over all prime ideals $\mathfrak{r}$ of $R$ such that $\operatorname{dim}(R / \mathfrak{r})=\operatorname{dim}(R)$. It follows that the only term in the $\operatorname{sum}$ is $\operatorname{len}\left(R_{\mathfrak{p}}\right) e(R / \mathfrak{p})$. Since $R$ is equidimensional, this implies that $\mathfrak{p}$ is the unique minimal prime of $R$.

If $e(R / \mathfrak{p})=1$ and $\mathfrak{p}$ is the unique minimal prime of $R$

$$
e(R)=\sum_{\mathfrak{r}} \operatorname{len}\left(R_{\mathfrak{r}}\right) e(R / \mathfrak{r})=\operatorname{len}\left(R_{\mathfrak{p}}\right) e(R / \mathfrak{p})=\operatorname{len}\left(R_{\mathfrak{p}}\right)=e\left(R_{\mathfrak{p}}\right)
$$

where the sum is taken over all prime ideals $\mathfrak{r}$ of $R$ such that $\operatorname{dim}(R / \mathfrak{r})=\operatorname{dim}(R)$. If $e\left(R_{\mathfrak{p}}\right)=e(R)$ and $\mathfrak{p}$ is the unique minimal prime of $R$, then a similar computation shows that $e(R / \mathfrak{p})=1$.

(b) If $R / \mathfrak{p}$ is unmixed, then [4] Theorem 6.8 implies that $e(R / \mathfrak{p})=1$ if and only if $R / \mathfrak{p}$ is regular.

A second indication that the condition $e\left(R_{\mathfrak{p}}\right)=e(R)$ is rather strong is supplied by the following lemma. First, we recall several notions. If $\mathfrak{a}$ and $\mathfrak{b}$ are ideals such that $\mathfrak{b} \subseteq \mathfrak{a}$, then $\mathfrak{b}$ is a reduction of $\mathfrak{a}$ if there exists a positive integer $n$ such that $\mathfrak{a}^{n+1}=\mathfrak{b} \mathfrak{a}^{n}$. In this case, $\mathfrak{b}$ is a minimal reduction of $\mathfrak{a}$ if it is minimal among all 
reductions of $\mathfrak{a}$ with respect to inclusion. If $(R, \mathfrak{m})$ is a Noetherian local ring with an ideal $\mathfrak{a}$, the Rees algebra of $\mathfrak{a}$ is the graded ring

$$
R[\mathfrak{a} t]=\bigoplus_{n=0}^{\infty} \mathfrak{a}^{n} t .
$$

The special fibre of the Rees algebra $R[\mathfrak{a} t]$ is the ring

$$
F(\mathfrak{a})=R[\mathfrak{a} t] \otimes_{R} R / \mathfrak{m} .
$$

The Krull dimension of $F(\mathfrak{a})$ is the analytic spread of $\mathfrak{a}$ and is denoted $s(\mathfrak{a})$. If the residue field of $R$ is infinite, then $s(\mathfrak{a})$ is the least number of elements required to generate a reduction ideal of $\mathfrak{a}$.

Lemma 3.3 (4] Theorem 20.9). Let $R$ be a quasi-unmixed local ring and let $\mathfrak{p}$ be a prime ideal of $R$ for which $R / \mathfrak{p}$ is regular. Then the following conditions are equivalent.

1. $e(R)=e\left(R_{\mathfrak{p}}\right)$,

2. ht $(\mathfrak{p})=s(\mathfrak{p})$.

Below, we indicate examples which show that the assumption " $R / \mathfrak{p}$ is regular" is essential for both implications.

Proposition 3.4. Assume that $(R, \mathfrak{m})$ is a quasi-unmixed local ring of dimensiond with prime ideals $\mathfrak{p}$ and $\mathfrak{q}$ such that $\sqrt{\mathfrak{p}+\mathfrak{q}}=\mathfrak{m}, R / \mathfrak{p}$ is regular and $e\left(R_{\mathfrak{p}}\right)=e(R)$. Then $\operatorname{dim}(R / \mathfrak{p})+\operatorname{dim}(R / \mathfrak{q}) \leq d$.

Proof. As in the proof of Theorem 2.1 we may assume that $R$ has infinite residue field by passing to the $\operatorname{ring} R(X)=R[X]_{\mathfrak{m}[X]}$. We note that $R(X)$ is quasi-unmixed and $R(X) / \mathfrak{p} R(X)$ is regular.

By Lemma 3.3. our assumptions imply that $s(\mathfrak{p})=\mathrm{ht}(\mathfrak{p})$. Since the residue field of $R$ is infinite, $\mathfrak{p}$ contains a sequence $y_{1}, \ldots, y_{i}$ which generate a minimal reduction of $\mathfrak{p}$ where $i=\mathrm{ht}(\mathfrak{p})$. Since $\sqrt{\mathfrak{p}+\mathfrak{q}}=\mathfrak{m}$, we see that $\mathfrak{q}$ is an ideal of definition for $R / \mathfrak{p}$ and therefore $\mathfrak{q}$ contains a system of parameters $z_{1}, \ldots, z_{j}$ for $R / \mathfrak{p}$. In particular $j=\operatorname{dim}(R / \mathfrak{p})$. We claim that $y_{1}, \ldots, y_{i}, z_{1}, \ldots, z_{j}$ is a system of parameters for $R$. Since $i+j=\operatorname{ht}(\mathfrak{p})+\operatorname{dim}(R / \mathfrak{p})=\operatorname{dim}(R)$, the sequence has the correct length and we need only check that the sequence generates an m-primary ideal. We compute

$$
\sqrt{(\mathbf{y}, \mathbf{z}) R}=\sqrt{\sqrt{(\mathbf{y}) R}+\sqrt{(\mathbf{z}) R}}=\sqrt{\mathfrak{p}+\sqrt{(\mathbf{z}) R}} .
$$

The fact that $\mathbf{z}$ is a system of parameters for $R / \mathfrak{p}$ implies that the only prime ideal of $R$ containing $\mathfrak{p}$ and $\mathbf{z}$ is $\mathfrak{m}$, as desired.

To prove the result, it suffices to show that $i \geq \operatorname{dim}(R / \mathfrak{q})$, as this will show that $\operatorname{dim}(R)=i+j \geq \operatorname{dim}(R / \mathfrak{q})+\operatorname{dim}(R / \mathfrak{p})$. In the $\operatorname{ring} R / \mathfrak{q}$, the images of $\mathbf{y}$ generate an ideal which is primary to $\mathfrak{m} / \mathfrak{q}$ since $\sqrt{(\mathbf{y}) R}=\mathfrak{p}$. Since $\operatorname{dim}(R / \mathfrak{q})$ is the least integer $l$ such that an ideal primary to the maximal ideal of $R / \mathfrak{q}$ can be generated by $l$ elements, we are done.

This result may be more interesting than it appears, as we hope to use de Jong's theory of regular alterations to reduce a number of general cases to the case where $R / \mathfrak{p}$ is regular. 


\section{EXAMPLES}

The following examples show that many of the assumptions we place on the local ring $R$ and the prime ideals $\mathfrak{p}$ and $\mathfrak{q}$ in Conjectures 1.2 and 1.3 may not be relaxed.

Example 4.1. If we do not require $R$ to be regular, then Conjecture 1.2 does not hold. Let $k$ be a field and let $R=k \llbracket X, Y, Z \rrbracket /\left(X^{2}-Y Z\right)=k \llbracket x, y, z \rrbracket$. Then $R$ is a complete intersection of dimension 2 , but is not regular. Let $\mathfrak{p}=(x, z)$ and $\mathfrak{q}=$ $(x, y)$ which are prime ideals in $R$ such that $\mathfrak{p}+\mathfrak{q}=\mathfrak{m}$ and $\operatorname{dim}(R / \mathfrak{p})+\operatorname{dim}(R / \mathfrak{q})=$ $2=\operatorname{dim}(R)$. However, $X \in \mathfrak{p} \cap \mathfrak{q}$ so that $\mathfrak{p} \cap \mathfrak{q} \nsubseteq \mathfrak{m}^{2}$. It is straightforward to verify that $\mathfrak{p}^{(2)}=(z)$ so that $\mathfrak{p}^{(2)} \cap \mathfrak{q}=(x z, y z)$.

Example 4.2. If we do not require $\sqrt{\mathfrak{p}+\mathfrak{q}}=\mathfrak{m}$, then Conjecture 1.2 does not hold. Let $R=k \llbracket X, Y \rrbracket$ and $\mathfrak{p}=\mathfrak{q}=(X)$. Then $R$ is regular and $\operatorname{dim}(R / \mathfrak{p})+\operatorname{dim}(R / \mathfrak{q})=$ $2=\operatorname{dim}(R)$. However, $\mathfrak{p} \cap \mathfrak{q}=(X) \nsubseteq \mathfrak{m}^{2}$.

Example 4.3. If we do not require that $\operatorname{dim}(R / \mathfrak{p})+\operatorname{dim}(R / \mathfrak{q})=\operatorname{dim}(R)$, then Conjecture 1.2 does not hold. Let $R=k \llbracket X \rrbracket$ and $\mathfrak{p}=\mathfrak{q}=(X)$. Then $R$ is regular and $\mathfrak{p}+\mathfrak{q}=\mathfrak{m}$. However, $\mathfrak{p} \cap \mathfrak{q}=(X) \nsubseteq \mathfrak{m}^{2}$.

Example 4.4. If we do not require that $e\left(R_{\mathfrak{p}}\right)=e(R)$, then Conjecture 1.3 does not hold. Let $k$ be a field, $R=k \llbracket X, Y, Z, W \rrbracket /(X Y-Z W)=k \llbracket x, y, z, w \rrbracket$ with $\mathfrak{p}=(x, z) R$ and $\mathfrak{q}=(y, w) R$. Then $e(R)=2>1=e\left(R_{\mathfrak{p}}\right)$ and $\operatorname{dim}(R / \mathfrak{p})+$ $\operatorname{dim}(R / \mathfrak{q})=4>3=\operatorname{dim}(R)$.

Example 4.5. If we do not require that $\sqrt{\mathfrak{p}+\mathfrak{q}}=\mathfrak{m}$, then Conjecture 1.3 does not hold. Let $R=k \llbracket X \rrbracket$ and $\mathfrak{p}=\mathfrak{q}=(0)$. Then $e\left(R_{\mathfrak{p}}\right)=e(R)$ and $\operatorname{dim}(R / \mathfrak{p})+$ $\operatorname{dim}(R / \mathfrak{q})=2>\operatorname{dim}(R)$.

Example 4.6. If we do not require that $R$ is at least equidimensional, then Conjecture 1.3 does not hold. Let $R=k \llbracket X \rrbracket \times_{k} k \llbracket Y, Z \rrbracket$. That is, in the diagram

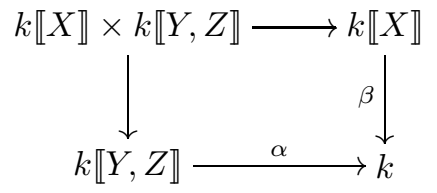

$R=\{(a, b) \in A \times B: \alpha(a)=\beta(b)\}$. Let $\mathfrak{p}=((X, 0)) R$ and $\mathfrak{q}=((0, Y),(0, Z)) R$. It is straightforward to verify the following facts: (i) $R$ is a local ring with maximal ideal $\mathfrak{m}=(X) \times(Y, Z)$; (ii) $\operatorname{dim}(R)=2$; (iii) $e\left(R_{\mathfrak{p}}\right)=e\left(R_{\mathfrak{q}}\right)=e(R)=1$; and (iv) $\mathfrak{p}+\mathfrak{q}=\mathfrak{m}$. However, $\operatorname{dim}(R / \mathfrak{p})+\operatorname{dim}(R / \mathfrak{q})=3>2=\operatorname{dim}(R)$. We should note, as this pertains to Conjecture 1.4 that both $\mathfrak{p}$ and $\mathfrak{q}$ have infinite projective dimension.

With Conjectures 1.3 and 1.4 in mind, one might hope that there is a nice relation between the conditions " $e\left(A_{\mathfrak{p}}\right)=e(A)$ " and "p has finite projective dimension". The following examples show that neither of these conditions implies the other.

Example 4.7. Let $R=k \llbracket X, Y, Z, W \rrbracket /\left(X^{2}-Y Z\right)=k \llbracket x, y, z, w \rrbracket$ with $\mathfrak{p}=(x, y, z)$. Then $\mathfrak{p}$ has infinite projective dimension and $e(R)=2=e\left(R_{\mathfrak{p}}\right)$. Notice that $R$ is a domain and $R / \mathfrak{p}$ is regular.

Example 4.8. Let $R=k \llbracket X, Y, Z, W \rrbracket /\left(X^{2}-Y Z\right)$ with $\mathfrak{p}=(0)$. Then $R / \mathfrak{p}$ is free (and therefore has finite projective dimension) and $e(R)=2>1=e\left(R_{\mathfrak{p}}\right)$. 
We notice that Example 4.8 is not as satisfying as Example 4.7, in the sense that $R / \mathfrak{p}$ is not regular. We were attempting to find a complete local domain $R$ with prime ideal $\mathfrak{p}$ satisfying the following conditions: (i) $R / \mathfrak{p}$ is regular, (ii) $\mathfrak{p}$ has finite projective dimension, and (iii) $e(R)>e\left(R_{\mathfrak{p}}\right)$. We have already seen that these conditions are quite restrictive. In fact, such an example does not exist, as we see in the following.

Lemma 4.9. Let $(R, \mathfrak{m})$ be a local ring and $M$ a finitely generated $R$-module. Let $F_{\bullet} \rightarrow M$ be an $R$-free resolution of $M$ and assume that $\mathbf{x}=x_{1}, \ldots, x_{i} \in R$ is a sequence which is regular on $M$. Let $K_{\bullet}=K_{\bullet}(\mathbf{x})$ denote the Koszul complex of $\mathbf{x}$ on $R$. Then $F_{\bullet} \otimes_{R} K_{\bullet}$ is a free resolution of $M / \mathbf{x} M$.

Proof. The fact that $K_{\bullet}\left(x_{1}, \ldots, x_{i}\right)=K_{\bullet}\left(x_{1}\right) \otimes K_{\bullet}\left(x_{2}, \ldots, x_{i}\right)$ allows us to assume that $i=1$. In this case, $K_{\bullet}$ is exactly

$$
0 \rightarrow R \stackrel{\cdot x}{\rightarrow} R \rightarrow 0
$$

We apply the mapping cone and the long exact sequence of homology to give the desired result.

Lemma 4.10. Assume that $(R, \mathfrak{m})$ is a local Noetherian ring with prime ideal $\mathfrak{p}$ of finite projective dimension. Then $R_{\mathfrak{p}}$ is a regular local ring. In particular, $\mathfrak{p}$ is locally a complete intersection.

Proof. A finite free resolution of $R / \mathfrak{p}$ over $R$ localizes to a finite free resolution of $(R / \mathfrak{p})_{\mathfrak{p}}$ over $R_{\mathfrak{p}}$. Now apply the theorem of Auslander, Buchsbaum and Serre to see that $R_{\mathfrak{p}}$ is regular.

Proposition 4.11. Let $(R, \mathfrak{m})$ be a local ring of dimension $d$ with prime ideal $\mathfrak{p}$ such that $\mathfrak{p}$ has finite projective dimension and let $\hat{R}$ denote the completion of $R$. Assume that one of the following holds:

1. $R / \mathfrak{p}$ is regular.

2. $R$ is unmixed and $e\left(R_{\mathfrak{p}}\right)=e(R)$.

Then $R$ is regular. (In particular, $e\left(R_{\mathfrak{p}}\right)=1=e(R)$ and every prime ideal has finite projective dimension.)

Proof. 1. Assume that $(R, \mathfrak{m})$ is a local ring with prime ideal $\mathfrak{p}$ such that $R / \mathfrak{p}$ is regular and $\mathfrak{p}$ has finite projective dimension. Let $F_{\bullet} \rightarrow R / \mathfrak{p}$ be a minimal, finite $R$-free resolution of $R / \mathfrak{p}$. Since $R / \mathfrak{p}$ is regular, let $\mathbf{x}=x_{1}, \ldots, x_{i} \in R$ be a sequence which forms a regular system of parameters of $R / \mathfrak{p}$. Let $K_{\bullet}=K_{\bullet}(\mathbf{x})$ denote the Koszul complex of $\mathbf{x}$ on $R$, so that, by Lemma 4.9, $F_{\bullet} \otimes_{R} K_{\bullet}$ is a finite, $R$-free resolution of $(R / \mathfrak{p}) /(\mathbf{x} R / \mathfrak{p})=R / \mathfrak{m}$. The theorem of Auslander, Buchsbaum and Serre implies that $R$ is regular.

2. Under our assumptions, $R$ is regular if and only if $e(R)=1$. By Lemma 4.10. $R_{\mathfrak{p}}$ is regular so that $e(R)=e\left(R_{\mathfrak{p}}\right)=1$, as desired.

The following example shows that the requirement of equidimensionality in necessary for the inequality $e\left(R_{\mathfrak{p}}\right) \leq e(R)$ to hold.

Example 4.12. Let $k$ be a field and $R=k \llbracket X \rrbracket \times_{k} k \llbracket Y \rrbracket /\left(Y^{n}\right)$ where $n>1$, as in Example 4.6. Then $R$ is a local ring with maximal ideal $\mathfrak{m}=(X) \times(Y), \operatorname{dim}(R)=1$, $e(R)=1$, and the ideal $\mathfrak{p}=(0) \times(Y)$ is a prime such that $e\left(R_{\mathfrak{p}}\right)=n>1=e(R)$.

The following examples show that if $R / \mathfrak{p}$ is not regular, neither implication of Lemma 3.3 holds. Note that each example is quasi-unmixed. 
Example 4.13. Let $R=k \llbracket X, Y, Z, U, V, W \rrbracket$ and $\mathfrak{p}$ be the prime ideal generated by the size two minors of the generic matrix

$$
\left(\begin{array}{ccc}
X & Y & Z \\
U & V & W
\end{array}\right)
$$

Since $R$ is regular, $e(R)=1=e\left(R_{\mathfrak{p}}\right)$. However, ht $(\mathfrak{p})=2$ and $s(\mathfrak{p})=3$.

Example 4.14. Let $R=k \llbracket X, Y, Z, W \rrbracket /(X Y-Z W)$ with $\mathfrak{p}=(0) R$. Then ht $(\mathfrak{p})=$ $0=s(\mathfrak{p})$. However, $e(R)=2$ and $e\left(R_{\mathfrak{p}}\right)=1$.

\section{REFERENCES}

1. Pierre Berthelot, Altérations de variétés algébriques (d'après A. J. de Jong), Astérisque (1997), no. 241, Exp. No. 815, 5, 273-311, Séminaire Bourbaki, Vol. 1995/96. MR 98m:14021

2. A. J. de Jong, Smoothness, semi-stability and alterations, Inst. Hautes Études Sci. Publ. Math. (1996), no. 83, 51-93. MR 98e:14011

3. Henri Gillet and Christophe Soulé, K-théorie et nullité des multiplicités d'intersection, C. R. Acad. Sci. Paris Sér. I Math. 300 (1985), no. 3, 71-74. MR 86k:13027

4. M. Herrmann, S. Ikeda, and U. Orbanz, Equimultiplicity and blowing up, Springer-Verlag, Berlin, 1988, An algebraic study, With an appendix by B. Moonen. MR 89g:13012

5. Bernd Herzog, On the Macaulayfication of local rings, J. Algebra 67 (1980), no. 2, 305-317. MR 82e:13029

6. M. Hochster, Nonnegativity of intersection multiplicities in ramified regular local rings following Gabber/De Jong/Berthelot, unpublished notes.

7. Kazuhiko Kurano and Paul C. Roberts, The positivity of intersection multiplicities and symbolic powers of prime ideals, Compositio Math. 122 (2000), no. 2, 165-182. MR 2001g:13031

8. Christer Lech, Inequalities related to certain couples of local rings, Acta Math. 112 (1964), 69-89. MR 28:5080

9. Hideyuki Matsumura, Commutative ring theory, Cambridge University Press, Cambridge, 1986, Translated from the Japanese by M. Reid. MR 88h:13001

10. C. Peskine and L. Szpiro, Dimension projective finie et cohomologie locale. Applications à la démonstration de conjectures de M. Auslander, H. Bass et A. Grothendieck, Inst. Hautes Études Sci. Publ. Math. (1973), no. 42, 47-119. MR 51:10330

11. Paul Roberts, The vanishing of intersection multiplicities of perfect complexes, Bull. Amer. Math. Soc. (N.S.) 13 (1985), no. 2, 127-130. MR 87c:13030

12. Paul C. Roberts, Recent developments on Serre's multiplicity conjectures: Gabber's proof of the nonnegativity conjecture, Enseign. Math. (2) 44 (1998), no. 3-4, 305-324. MR 2000c:13031

13. Jean-Pierre Serre, Algèbre locale. Multiplicités, Springer-Verlag, Berlin, 1965. MR 34:1352

Department of Mathematics, University of Utah, 155 S. 1400 E., Salt Lake City, UTAн 84112-0090

Current address: Department of Mathematics, University of Illinois, 273 Altgeld Hall, 1409

W. Green St., Urbana, Illinois 61801

E-mail address: ssather@math.uiuc.edu 